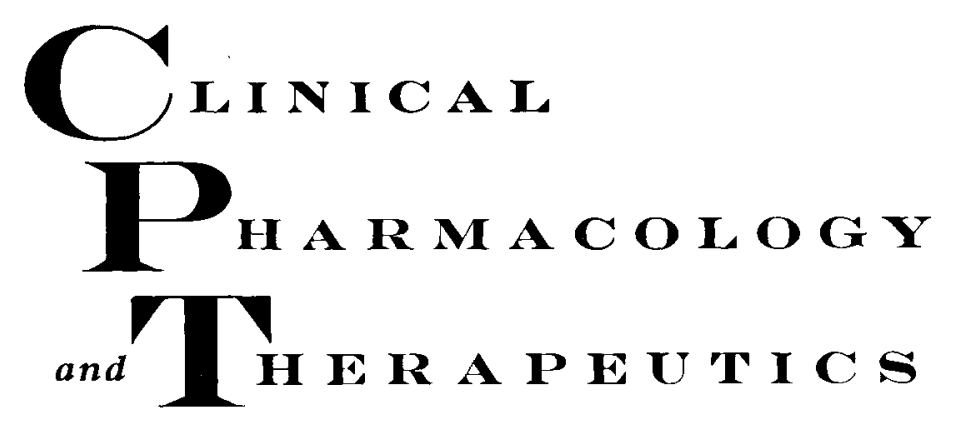

volume 5 number 2

March-April, 1964

\title{
Editorial
}

\section{Adverse reactions to drugs: Should we have formal programs for reporting them?}

Edward A. Carr, Jr., M.D. Ann Arbor, Mich.

Departments of Pharmacology and Internal Medicine, University of Michigan School of Medicine

The strong right arm of therapeutics has made such benefits available to patients in recent years that few would wish to return to the past. Those who decry treatment with modern drugs should go to some area of the world where these drugs are not available and see how it is to live (or more often die) without them. But our patients still run the risk of falling into another sinister grip-serious adverse reactions to the drugs we use. There has been a tendency for many physicians to accept too eagerly the immediate benefits of modern drugs, while a sour minority has stressed almost exclusively their adverse effects. It is time the right hand knew what the left hand is doing, and vice versa.

In some medical centers, a formal program for the detection and reporting of adverse reactions to drugs has been instituted.

Received for publication April 2, 1963.
These programs are designed to collect detailed information on every adverse reaction that occurs within the medical center and make this information available to all physicians associated with the center. The question is: Do such programs really accomplish any good? If they do, what factors contribute most to their success?

The chief value of such programs is not that they "give us an over-all picture," "provide a broad survey of the problem," and so forth. In many aspects of sociology and science during the past ten years, we have been "given over-all pictures" and "provided with broad surveys" to such a point that no one can digest the data. The chief value of a program for reporting drug reactions is that it makes everyone involved alert to the dangers of modern therapy and this alertness is based on "close to home" occurrences that really impress themselves on the minds of those associated with the medical center in question. Therefore, 
prompt and practical solutions to some of the problems discovered can often be worked out by the staff. The reports, if presented at reasonable intervals to the entire staff, bring to the attention of each physician new dangers that have arisen with new remedies. Thus, when the administration of a certain intravenous vitamin $K$ preparation was found to be followed by a severe anaphylactoid reaction in two patients, this information was immediately made available to the Pharmacy and Therapy Committee, the use of the preparation was immediately discontinued until a method for its safe administration could be worked out, and the staff was notified. In the field of drug reactions, as in contagious diseases, it is often desirable for the physician in any given area to know "what is going around this year." Another value of such programs is their "seed value"; young physicians taking part in them are likely to develop an interest in clinical pharmacology and will perhaps institute similar programs in other hospitals and medical centers in future years.

There are two major disadvantages to such programs. First, there is already a huge superstructure of committees, reports, administrative make-work, etc., piled upon the practicing physician. Like his brother, the active scientific investigator, he already spends far too much time with such nonsense. Therefore, no additional program involving forms, reports, etc., can be tolerated unless it makes a very real contribution to medicine. Second, the constant collection and reporting of adverse reactions to drugs is likely to have the same effect upon the local attorneys as a brisk hemorrhage into the water has upon nearby sharks.

The first objection is more easily met than then second. A program for reporting adverse drug reactions probably can be carried out without cumbersome paper work and probably contributes enough to the safety of the patient to justify it. The second objection is a more serious one. On one occasion, I gave a talk on drug reac- tions before a county medical society and was surprised to find that my appearance and talk were given advance publicity which scrupulously omitted the title of the talk. I could easily understand why my appearance in town would not necessarily deserve any type of advertisement at all, but I could not understand why, once the arrangement committee had decided upon advance publicity, the title of the talk should be omitted. Upon inquiry, I was informed that the subject of adverse reactions to drugs had made everyone so jittery and sensitive that it was deemed best to keep the phrase "adverse reactions to drugs" entirely out of the local newspaper. This may represent an extreme case, but it does show the general reaction engendered by lawyers in the minds of most doctors. This state of affairs will continue until such time as the courts finally begin to dispense more even justice in professional liability suits. If this day ever dawns, then programs for reporting adverse drug reactions will be most helpful. They will serve to protect the physician who exercises reasonable caution, in that the courts will give directed verdicts in favor of physicians who can demonstrate that they did show reasonable caution. Such programs will serve to restrict liability to those who are deservedly liable. If a series of reasonable standard steps to decrease the incidence of drug reactions is established, then the physician who takes these standard precautions deserves to be protected by the court just as much as the physician who ignores them deserves to be held liable. At the present time, of course, the legal philosophy is, "If you don't take precautions, you are, of course, liable; if you do take precautions, you are liable anyway."

Thus, the development of programs for reporting adverse drug reactions in large medical centers and other hospitals seems to have more advantages than disadvantages, although the last objection discussed does represent a most serious and potentially fatal disadvantage.

What are the factors that make such pro- 
grams feasible and successful? First, one must realize that it is a waste of time to pass out forms to the various wards of a hospital and ask that they be filled out whenever a drug reaction occurs. It is necessary to choose a "leg man," a physician who constantly makes the rounds of the hospital and regularly asks nurses, physicians, medical students, etc., about the occurrence of adverse reactions. Some sort of reward must be offered the man who takes on this burden. Surprisingly, committees often seem at a loss to devise a suitable reward. Over the last several thousand years a considerable body of evidence has accumulated suggesting that money is considered a rather acceptable reward in many fields of endeavor. If a resident or junior visiting man is assigned this task, he must be someone who haunts the wards of the hospital constantly and the hospital must see that he is paid for his pains.

Second, each report must be written on one standard form, not exceeding one page. The form in use at The University of Michigan Hospital provides for recording the following information: the suspected compound, disease under treatment, other pertinent conditions, trade name, composition, dosage form, manufacturer's lot number (if known), etc.; the patient's name, occupation, attending physician; a description of the reaction and the details of the dosage schedule (number of doses, total amount, date of first dose, time interval between last dose and onset of reaction, history of previous exposure, etc.), concurrent drug therapy; outcome and additional remarks.

There should be a standard procedure to provide for immediate recording of suspected cases of hypersensitivity on the front of the patient's chart. I know of one instance in which fatal penicillin anaphylaxis occurred after a patient had been transferred from one clinical service to another, because the allergy to penicillin that had been discovered on the first service was buried in the progress notes of the patient's thick chart and was not seen by those on the second service who wished to order penicillin for the patient. In another instance, a senior visiting man discovered that a patient was hypersensitive to aspirin and observed that no note had been made on the front of the patient's chart. $\mathrm{He}$ promptly called the house staff and medical students together in an adjoining room and pointed out the necessity of having such information on the front of the chart. While he was explaining this, a dose of aspirin was administered by a nurse on the ward. The patient died of the reaction.

Reports should be made to the staff at regular intervals but it is probably not advisable to monopolize every monthly staff meeting with such reports. Semiannual reports with supplementary emergency reports of serious individual problems, will keep the staff alert. There should, however, be an arrangement whereby the pharmacy can immediately stop all administration of a given agent within the hospital and immediately collect all outstanding supplies from the wards, if an emergency seems to dictate this. Such extreme decisions, should, of course, be rare.

There is no compelling reason to restrict such programs to large medical centers. Smaller hospitals may not have the services of a resident to walk the wards daily but one of the younger men starting in practice may be glad to have an opportunity to put in an appearance at the hospital every day and go to each of the clinical units. Such an individual, particularly if he has had training in clinical pharmacology, will soon become the local authority on drug reactions and may also be the logical man to assume organization and direction of the hospital's poison control center.

The administration of such a program should be sharply separated from disciplinary action. Certainly, in the vast majority of cases, reactions to drugs are misfortunes that hardly call for disciplinary action against a member of the staff. On the rare occasion that may call for such action, it would be advisable for those concerned with the drug reaction program to dissociate themselves from the judging, as they 
are already the principal witnesses. Any disciplinary action, if indicated, should be carried out according to the regular protocol of the hospital staff.

The matter of reporting reactions to any central bureau in Washington or elsewhere deserves thought. Provided the identity of patient and physician is strictly protected, such reporting may be useful in that it enables a collection of data from many centers to be made. Moreover, funds for paying for the program are available in certain instances. On the other hand, experience so far has suggested that the feedback from central bureaus is slow or nonexistent, and, to date, medical centers seem to learn most from the reports of reactions that they have collected themselves. As the new Food and Drug regulations require such reporting of reactions to investigative drugs, it is to be hoped that in the future a more effective method of centralized data collection and feedback to participating hospitals may be developed.

Finally, after all this information about adverse reactions to drugs is presented to staff members, what can they do about the problems described, anyway? After the left hand has been introduced to the right hand, are they simply to wring each other? Not at all. For example, the staff may decide that Drug A should be discontinued from hospital use entirely; fresh solutions of Drug B can no longer be made up on the wards because contamination is too easy and all preparations will have to be made up in the pharmacy; Drug $\mathrm{C}$ is too valuable to discontinue but the prevalence of reactions to it suggests that physicians place it on their mental list of drugs reserved for serious emergencies; Drug D is apparently causing trouble whenever it is given too rapidly intravenously and, therefore, it must be available only in dilute solutions; Drug $\mathrm{E}$ has been inculpated in a certain reaction but a review of the details suggests that the reaction was not due to $\mathbf{E}$ at all, and the drug is acquitted; Drug $F$ causes a reaction that is usually preceded by a day or two of itching and if the staff is alert for this sign and discontinues the drug at once, the subsequent reaction will be much less severe, and so on. If the medical center has a large staff, time will be saved if the Pharmacy and Therapy Committee makes preliminary decisions. These can subsequently be presented to the whole staff for its approval.

In summary, programs for reporting adverse reactions to drugs are not free of objections but they should be instituted because they seem to do considerably more good than harm. If this seems too weak a reason, consider this: It is the same reason that justifies giving drugs in the first place. 